\title{
La contabilidad analítica y la medida de la eficiencia en los servicios públicos
}

Santiago Fuentes Vega *

\section{Introducción}

Podemos considerar a la Contabilidad, extractando la definición que de ella da el profesor CaÑIBano como una ciencia de naturaleza económica cuyo objeto es conocer la realidad también económica de un ente en términos cuantitativos, para elaborar información útil para las necesidades financieras externas, y para las de planificación y control internas del ente en cuestión.

En este concepto se plasman y ponen de manifiesto dos hechos:

a) El objeto de la contabilidad es conocer la realidad económica.

b) La finalidad de la contabilidad es dar información útil.

En una primera aproximación sencilla y por tanto fácilmente comprensible, si bien como toda simplificación susceptible de ser matizada, se podría señalar que la información hacia el exterior nos la facilitará la contabilidad financiera, en tanto que la información útil para los propios gestores del ente, la información necesaria para la toma de decisiones, será suministrada por la contabilidad analítica.

La contabilidad analítica como ciencia para conocer la realidad económica interna de un ente, con la finalidad de suministrar información útil a los gestores para tomar decisiones que mejoren la gestión, es necesaria, tanto en el ámbito público, como en el privado, aunque en este trabajo voy a referirme exclusivamente a la gestión públića.

De manera sencilla y útil a los efectos de la exposición se puede entender por Sector Público la parte de la econonía nacional situada bajo la autoridad de los poderes públicos. El
Sector Público así entendido engloba dos grandes grupos de entidades:

Administraciones Públicas

Empresas Públicas.

El subsector Administraciones Públicas es definido por el Sistema Europeo de Cuentas Económicas Integradas como: "aquel que comprende todas las unidades institucionales que, a título de función principal, producen servicios no destinados a la venta para la colectividad y/o efectúan operaciones de redistribución de la renta y de la riqueza nacional. Los recursos principales de estas unidades proceden de pagos obligatorios efectuados por unidades pertenecientes a otros sectores y recibidos directa o indirectamente". Este subsector, pues, tendrá una gestión pública y estará sometido a contabilidad pública. Su característica primordial, de la que derivan muchos de los problemas que, tanto en el ámbito de la gestión, como en el de la contabilidad, plantea, es el hecho mismo de actuar sin ánimo de obtener beneficio. Es precisamente esta carencia de ánimo de lucro la que explica bastantes de los avatares experimentados por la Contabilidad Pública en el tiempo.

\section{La contabilidad en la Administración Pública}

\subsection{La evolución de la contabilidad pública}

La contabilidad en el ámbito público ha sido una materia, más que olvidada, subordinada a la teoría de la Hacienda Pública, y sobre todo a la institución presupuestaria. 
El presupuesto que en la empresa privada ha sido un simple, aunque importante, plan de acción meramente indicativo, en la Administración Pública ha sido el instrumento fundamental de la política económica. Era y es un estado de origen y aplicación de fondos, pero al ser aprobado anualmente por el Parlamento en forma de Ley, es de obligado cumplimiento y de una enorme rigidez, ya que incluye gastos limitativos que están vinculados a los conceptos en los que son aprobados. El presupuesto tradicional daba respuesta al ideario clásico, ideario que se plasmaba en principios que en el campo de la contabilidad eran los conocidos de: presupuesto bruto, unidad de caja, especificación y ejercicio cerrado, que eran la traslación de los principios político-presupuestarios al ámbito contable.

La contabilidad pública era, pues, hasta época muy reciente una contabilidad estrictamente presupuestaria, más aún, únicamente de ejecución presupuestaria, en la que la información que se ofrecía mostraba como era gastado el presupuesto anual y servía fundamentalmente a los efectos del control a realizar, tanto por los órganos de verificación internos, como, sobre todo, por los externos.

Esta concepción de la contabilidad tradicional existente en la mayoría de los países comienza a quebrar cuando se aprecia la necesidad de disponer de información útil para conocer la realidad económico-patrimonial de las Administraciones. El momento en el que esa necesidad se siente en los distintos países varía en el tiempo, porque no todos la experimentan en el mismo momento. Concretamente en España es en la década de los setenta cuando se comienza a exigir de la Contabilidad que sirva para algo más que para dar información de la ejecución presupuestaría.

La plasmación legal de ese cambio en la forma de entender los fines de la Contabilidad se produce con la aprobación en 1977 de la L.G.P. cuyo art. 124 establece como fines de la contabilidad, entre otros:

b) Conocer el movimiento y la situación del Tesoro.

c) Reflejar las variaciones, composición y situación del Patrimonio del Estado.

e) Facilitar los datos y demás antecedentes que sean precisos para la confección de las cuentas económicas del Sector público y las nacionales de España.

f) Rendir la información económica y financiera que sea necesaria para la toma de decisiones, tanto en el orden político como en el de gestión.

Interesa llamar la atención sobre el vuelco cualitativo que en materia de contabilidad suponía este artículo y ello porque claramente introducía como metas a alcanzar, no sólo la tradicional de registro de la ejecución del presupuesto, sino la de posibilitar el conocimiento de la situación de la tesorería y del
Patrimonio del Estado y sus movimientos y lo que era más importante por los efectos sobre la modernización de la Administración Pública, la de facilitar la información económica-financiera necesaria para la toma de decisiones, tanto en el orden político como en el de la gestión.

El mismo impulso normalizador experimentado en la contabilidad empresarial, impulso que dio lugar a la aparición de los distintos Planes de Contabilidad y concretamente en España al Plan de 1973, se sintió en el Sector Público, si bien como suele ser habitual, con un cierto retraso en el tiempo.

La clara insuficiencia de la información que suministraba la Contabilidad tradicional y la necesidad de que la suministrada por los diferentes servicios u organismos fuese homogénea de forma que se pudiesen agregar, o consolidar las cuentas de los diferentes entes sometidos a contabilidad pública, explican la aparición de Planes de Contabilidad Pública y justifican el esfuerzo normalizador realizado en este ámbito. En España y por Orden del Ministerio de Economía y Hacienda de 14 de octubre de 1981 se aprobó el primer Plan General de Contabilidad Pública.

El proceso de normalización contable que ha tenido lugar en el ámbito privado y que dio lugar al desarrollo y aparición de una serie de principios formales contables, se ha producido en el ámbito público y ha llevado a la elaboración de unos principios contables que permiten hablar de una especie de marco conceptual básico para la contabilidad pública.

El diseño de este marco conceptual responde a una tendencia experimentada en muchos países y organizaciones internacionales, con la que se pretende normalizar la información suministrada por diferentes instituciones Públicas de manera que sea homogénea y comparable.

Tanto el marco conceptual que configuran los documentos aludidos, en especial el primero relativo a los principios contables públicos, como los avances producidos en materia de contabilidad, de los que los citados documentos no son más que un adecuado reflejo, así como la aprobación en 1990 del Nuevo Plan General de Contabilidad para la empresa española, hacían necesaria la aprobación de un nuevo Plan de Contabilidad Pública lo que se produce por Orden Ministerial de 6 de mayo de 1994.

El nuevo Plan General de Contabilidad Pública de 1994 comenzó a ser aplicado en el año 1995 y con él se pretende atender las necesidades de información a las que da respuesta la contabilidad financiera.

\subsection{Los sistemas de información contable}

Un plan contable es un modelo de información y control de una realidad económica concreta. 
Este modelo, o plan, ha de servir para suministrar información de la situación patrimonial de la unidad a la que se aplique, así como de las corrientes o flujos que puedan provocar alteraciones de dicha situación patrimonial. Esto, con ser mucho, no puede considerarse suficiente en el ámbito público, por la enorme cantidad y heterogeneidad de personas y entes que configuran ese sector, lo que hace, pero sobre todo hacía, difícil la agregación o consolidación de sus cifras y sobre todo por el gran volumen de operaciones que alguno de esos entes realiza lo que hacía difícil el tratamiento de dichas operaciones y la obtención de datos agregados en tiempo adecuado. Me atrevería a señalar que, sin el enorme desarrollo de la informática, no se hubiera podido dar solución a los problemas que presenta en el ámbito público esa heterogeneidad de entes y el volumen de operaciones que algunos realizan.

La respuesta al problema de suministro de información adecuada y suficiente, se trata de solucionar, en general, con la introducción de sistemas de información.

En España, concretamente, la puesta en funcionamiento de un sistema de información contable se produce en 1986. Es el sistema de información contable presupuestario SICOP. Dos son los datos que a la altura de 1986 permiten la introducción del SICOP:

- Un Plan de Contabilidad Pública.

- Un adecuado desarrollo informático.

El SICOP debe de ser considerado un conjunto de normas, procedimientos y utilidades para satisfacer las necesidades de información contable en el ámbito presupuestario para todos los interesados en ella. Se trata de un sistema mucho más complejo que la base informática que lo soporta y que el Plan Contable al que se acomoda la información que proporciona.

El SICOP permitió superar la mera visión presupuestaria y de tesorería de los entes del sector público, para considerar también aspectos patrimoniales y económicos. El tratamiento informático permitió la verificación sencilla de la compatibilidad de los datos contabilizados, su tratamiento en tiempo real y como consecuencia obligada, la rendición de las cuentas públicas en el plazo legal previsto, lo que supuso un gran avance.

La trascendencia de la implantación del SICOP ha sido enorme y puede que no se haya aún valorado en su totalidad. En cualquier caso comparto la opinión de quienes señalan que examinado el SICOP después de diez años de funcionamiento puede afirmarse que su implantación no supuso una mera reforma contable, sino una auténtica revolución tanto desde el punto de vista técnico como desde el funcional, no siendo desdeñable el cambio cultural que ha provocado en el ámbito administrativo. Creo sinceramente que la mayor renovación de la Administración en materia de gestión financiera la ha supuesto, sin duda, el funcionamiento normal del sistema de Información Contable Presupuestario.

Después de diez años de funcionamiento del sistema, en enero de este año ha comenzado a aplicarse una versión nueva el llamado SIC o Sistema de Información Contable. Lo primero que cabe señalar es que se trata de un sistema de información que engloba como su antecesor el equipo físico y los programas que lo soportan, las personas, las bases de datos, los manuales de trabajo, los impresos, la documentación explicativa y por supuesto los procedimientos a seguir para cada ocasión.

El SIC "2" tiene unas características generales muy ambiciosas que permiten calificarlo como:

- Completo

- Descentralizado

- Actualizado en tiempo real

- Flexible

- Seguro

Se trata además de un sistema preparado para la Administración del Estado. Con la misma filosofía para la Administración Institucional se puso en marcha en enero de 1995 el mismo sistema que ha venido también a sustituir al anterior de Información Contable para la Administración Institucional. (SICAI).

El SIC se configura como un conjunto de subsistemas relacionados entre sí y entre los que cabe distinguir:

- Subsistemas de información contable y de seguimiento y control presupuestario que pueden ser calificados de básicos.

- Subsistemas de información general para completar los anteriores y diseñados para conocer mejor la actividad del ente de que se trate y para facilitar su gestión.

Los subsistemas que hemos llamado básicos son los de:

- Contabilidad financiera.

- Ejecución del presupuesto de gastos.

- Ejecución del presupuesto de ingresos.

- Operaciones no presupuestarias.

- Operaciones comerciales.

- Tesorería.

- Interesados o terceros.

- Proyectos de gasto.

- Gastos con financiación afectada.

- Control de los remanentes de crédito.

Los demás subsistemas completan a éstos y permiten mejorar la información y ayudar al gestor de la entidad, entre ellos podemos señalar los de: 
- Justificantes de gasto.

- Inmovilizado no financiero.

- Préstamos concedidos.

- Control y gestión del endeudamiento.

- Contabilidad analítica.

\subsection{La contabilidad de gestión}

El gasto público tiene en la mayoría de los países un importante peso en relación con el P.I.B.. Este dato unido a las mayores exigencias ciudadanas tanto respecto a la calidad de los servicios que presta el Sector Público, como a la de la información sobre el gasto público, explican la preocupación creciente de los gestores por tomar decisiones acertadas para ser más eficientes en la utilización de los recursos escasos que se ponen a su disposición.

Conviene señalar que sin el proceso modernizador experimentado por las Administraciones Públicas de muchos países, no se hubiera producido el actual interés por la contabilidad de gestión en el ámbito de las citadas administraciones.

Consecuencia de las necesidades sentidas por los ciudadanos y del proceso modernizador de la Administración los gestores públicos necesitan cada vez en mayor medida disponer de datos que les permitan:

a) Valorar los actos derivados de la actividad que realizan.

b) Poder analizar los resultados de su explotación

c) Controlar y planificar su gestión.

d) Disponer de información fiable para la toma de decisiones.

Se hace preciso obtener información sobre las actividades de la organización, los productos que obtiene, los centros de producción y las funciones que realiza, para conocer con detalle el coste real de nuestros outputs. Cuanto se ha mencionado implica disponer de información sobre aspectos económicos valorables de la actividad realizada, es decir, conocer datos que sólo pueden ser facilitados por la contabilidad, pero no por la financiera, sino únicamente por la contabilidad interna, analítica o de costes.

Este es el momento en que nos encontramos. Se producen en la actualidad claros intentos por implantar un sistema de contabilidad analítica en las Administraciones Públicas. El primer paso, en ese largo camino, se inició en España con la aprobación en 1983 de la Orden Ministerial de 20 de septiembre que desarrolló el grupo 9, Contabilidad Analítica, del cuadro de cuentas correspondiente al Plan aprobado aquel mismo año. Actualmente y después de diversos trabajos más bien poco afortunados, que permitieron disponer de una experiencia necesa- ria en la materia, se trabaja en la implantación del proyecto de Contabilidad Analítica Normalizada de Organismos Autónomos (C.A.N.O.A.), al que luego me referiré.

3. La renovación

de la Administración Pública

\subsection{Gestión financiera y modernización}

Después del breve recorrido sobre la realidad histórica de la Contabilidad Pública en los últimos años, conviene examinar lo sucedido en la gestión financiera, porque la Contabilidad refleja esa gestión, pero para que ese reflejo sea fiel y útil debe acomodarse a sus necesidades.

El cambio experimentado en la gestión de lo público en los últimos años ha sido tan espectacular como el vivido por la contabilidad. La Administración todavía en el momento presente responde a un esquema de actuación muy tradicional y burocrático, con formas organizativas rígidas que no obedecen, en general, a criterios relacionados con la actividad o con el servicio prestado. Los gestores públicos aparecen preocupados por aplicar, de acuerdo con los procedimientos legalmente establecidos, los medios presupuestarios puestos a su alcance, antes que por la consecución de unos objetivos concretos, por lo demás no siempre claramente establecidos.

En este contexto, la contabilidad pública ha sido un instrumento para reflejar las operaciones de ejecución presupuestaria y ha estado volcada de una manera preponderante a facilitar los datos necesarios para el adecuado control legal de la forma en que son utilizados los fondos públicos incluidos en el presupuesto.

Este tipo tradicional de Administración es la respuesta lógica a una organización y una mentalidad muy generalizada en el siglo pasado e incluso a principios de este siglo. El Estado liberal organiza su Administración para que haciendo lo imprescindible, lo haga de la forma más anodina posible. Este ideario quebró por dos hechos hoy pacíficamente admitidos: de una parte, la percepción del fracaso del mercado como único y exclusivo regulador de la vida económica del país, al tiempo que por las exigencias ciudadanas tendentes a conseguir la implantación real del Estado de bienestar. El intervencionismo, pues, fue una consecuencia obligada de la asunción del nuevo ideario. El Estado se obliga, ya directamente, ya a través de múltiples subvenciones, a una intervención directa en la vida económica del país.

El crecimiento del gasto público en estas condiciones estaba asegurado. Y de hecho así sucedió en la mayoría de los países 
de la O.C.D.E., y por supuesto también en España, si bien con algunas diferencias en el tiempo.

Pero el gasto público realizado por las Administraciones no sólo ha cambiado en su tamaño, sino que ha variado en su estructura. Hemos asistido en los últimos años a un proceso de descentralización del gasto público, proceso caracterizado por la aparición de entes de diversa naturaleza que han adoptado nuevos modelos financieros buscando una mayor flexibilidad y autonomía de gestión, en aras de conseguir una mayor eficacia.

Esta búsqueda de una mayor eficacia, no sólo se producía por el hecho de que la Administración se enfrentase a un mayor volumen de recursos a gestionar, sino también por las críticas constantes y a veces justificadas sobre las deficiencias del modelo de gestión tradicional, modelo en el que la administración de los recursos públicos se hacía con criterios mucho más formales.

Es en este contexto en el que hay que plantear los intentos de renovación, modernización o como quiera que deseemos llamarlos, de la Administración, intentos que tratan de poner coto al aumento del volumen de gasto y al mismo tiempo tratan también de hacer a la Administración más eficaz y más eficiente porque se ha percibido claramente que dado que los recursos son escasos no se puede gestionar un importante volumen de ellos al margen de las más elementales consideraciones en relación con la eficiencia en la gestión. Se trata de pasar de una forma de funcionamiento excesivamente formal, a una gestión por objetivos orientada de continuo a la obtención de resultados, con obligación de rendir cuentas de los obtenidos.

En definitiva, con la modernización de la gestión pública se trata de conseguir lo que en términos de común aceptación podríamos llamar una buena gestión financiera, es decir, una gestión eficaz, eficiente y económica, y ello porque como he dicho se producen y se van a seguir produciendo demandas ciudadanas de más y mejores servicios públicos.

\subsection{Sistemas integrados de gestión}

La realidad que acabamos de examinar ha producido en la contabilidad pública un gran avance que ha supuesto pasar de reflejar la ejecución presupuestaria a mostrar la realidad económico-patrimonial y de ahí sin solución de continuidad a preocuparse por la aproximación al conocimiento interno de la gestión pública y con ello a intentar verificar en qué medida esa gestión es eficaz y eficiente.

Como momento que marca a nivel internacional el cambio a que me estoy refiriendo podríamos señalar el año de 1971 en que la ONU publica su Manual de Contabilidad del Gobierno. En dicho Manual de una manera ya clara se fijan como objetivos de la contabilidad pública, entre otros, ofrecer información de interés para los ciudadanos, útil para la planificación y direc- ción de la gestión pública y por tanto para quienes tienen que tomar decisiones y que al mismo tiempo permita realizar el análisis económico de la realidad sometida a control.

Esta forma de entender el objeto de la contabilidad pública se aproxima mucho a la que existe en la contabilidad empresarial. Con anterioridad se pensaba que la contabilidad empresarial tenía que estar diseñada para ofrecer información con carácter general (contabilidad financiera), y hacia el interior (contabilidad analítica), en tanto que la pública cumplía su objetivo al informar sobre la ejecución presupuestaria, y ello porque el presupuesto, aprobado por el Parlamento, y por tanto de obligado cumplimiento, se aplica a aquellas organizaciones cuya existencia, por definición, no se justifica por la búsqueda del beneficio, por lo que éste no puede ser utilizado para conocer y cuantificar los resultados. Esta limitación para la contabilidad pública ha dejado de tener sentido en la actualidad.

En la actualidad la contabilidad pública tiene que ofrecer al igual que lo hace la privada, información útil para mejor gestionar lo público, al no poder acudir para hacerlo a la medida de los beneficios, tiene que recurrir al conocimiento e identificación de los objetivos que justifican la existencia de las Administraciones Públicas. Conviene en este sentido recordar que si el objetivo casi único en la teoría de la Hacienda Clásica era la satisfacción de las necesidades públicas, los objetivos que la moderna teoría de la Hacienda señala a la actividad financiera de las citadas Administraciones son, al menos:

- Satisfacer las necesidades públicas

- Mejorar la distribución de la renta

- Propiciar el crecimiento estable de la economía

Para la consecución de estos objetivos las Administraciones Públicas realizan una actividad que se caracteriza fundamentalmente por:

- Producir bienes y servicios no destinados a la venta y realizar operaciones de redistribución de renta.

- Obtener sus recursos con pagos obligatorios (es decir, detraídos coactivamente) de particulares y empresas.

De estos dos hechos se deduce que en las Administraciones Públicas la correlación entre ambos juega justo en sentido inverso a aquel en el que lo hace en las empresas privadas, ya que su actividad viene determinada por las exigencias de los ciudadanos y para realizarla tienen que procurarse ingresos obtenidos coactivamente por el montante necesario para cubrirlas, en tanto que en la empresa privada que tiende a maximizar el beneficio será el importe de los ingresos que se puedan obtener el que condicionará la actividad a realizar. En la Administración, pues, se trata de maximizar las prestaciones por la vía de aumentar los gastos públicos o la eficacia de la actuación. 
En definitiva, para la empresa el saldo entre sus ingresos y gastos será el indicador básico para conocer el grado en que ha cumplido su objetivo: maximizar el beneficio, en tanto que para la Administración este saldo no es más que una magnitud intermedia que únicamente le señala si con los recursos corrientes obtenidos ha podido hacer frente a las actividades corrientes que tiene que realizar o, por el contrario, ha tenido que recurrir a otras fuentes de financiación.

El resultado en una Administración es complejo y cuando se habla de él no se hace referencia a un valor concreto, sino a una serie de cifras e indicadores que nos permiten una aproximación a ese dato fundamental menos precisa y por tanto más difícil de abordar. Para hablar de resultados en una Administración Pública, la ausencia de beneficio nos obliga a prestar atención a tres conceptos que se han hecho claves en los estudios de gestión pública en los últimos años: Eficacia, eficiencia y economía.

La eficacia se refiere a la capacidad de la organización para conseguir los objetivos que tiene señalados. Se trata de la relación entre los resultados obtenidos, medidos en unidades físicas o monetarias, y los objetivos establecidos. La eficiencia pone en relación los resultados obtenidos con los medios utilizados para obtenerlos, de manera que la mayor eficiencia posible se conseguirá cuando con unos medios dados se maximicen los resultados obtenidos, o cuando para obtener unos determinados resultados hayamos utilizado el menor número de medios.

Finalmente, la economía hace referencia únicamente a los medios y pone en relación los presupuestados con los realmente aplicados, de manera que cuando aquellos sean los estrictamente incluidos en el presupuesto, si éste está adecuadamente calculado, los medios utilizados lo habrán sido al menor coste previsto.

Pues bien, la posibilidad de evaluar adecuadamente la actividad de la Administración Pública pasa, conforme recomienda la GASB (Governmental Accounting Standars Board), en el segundo de sus documentos, por el señalamiento claro de indicadores que permitan conocer con exactitud a quienes tienen que tomar decisiones, y a los propios ciudadanos, la eficacia, eficiencia y economía con la que se gestiona.

Los indicadores han de ser cuantificables y medibles. Puede haber múltiples indicadores, tales como: indicadores de medios utilizados para realizar la actividad (indicadores de inputs); indicadores de los resultados obtenidos (indicadores de ouputs); indicadores de impacto a causa de los resultados obtenidos (indicadores de outcomes o impacto), indicadores del esfuerzo de gestión realizado (indicadores de gestión) que relacionan los medios utilizados para llevar a cabo la actividad y los resultados obtenidos.

Como puede comprobarse se trata de que con los diferentes indicadores se disponga de una información más útil sobre la gestión realizada por la entidad pública, información que resulta más completa que la que proporciona la cuenta de explotación y el balance que facilita la contabilidad financiera.

En definitiva, al hablar de los resultados en la Administración Pública no podemos hacer mención a los beneficios como sucede en el ámbito empresarial, sino que hemos de trabajar con los conceptos de eficacia, eficiencia y economía. La aproximación a estos términos ha de venir por la utilización en la Administración de sistemas integrados de gestión, sistemas que manejados con mayor soltura en el ámbito empresarial han sido adoptados en los últimos años por los gestores públicos con determinadas modificaciones. Como sistemas integrados de ges. tión podemos citar el presupuesto por programas y el presupuesto en base cero.

Las cuatro fases que componen el modelo de un presupues. to por programas representadas en la forma secuencial en que se producen son: planificación, presupuestación, ejecución y evaluación y control. Este modelo tiene unos rasgos muy característicos. En efecto, se trata de un proceso integrado en el que las distintas fases que lo componen se repiten sin fin, es más, el resultado de una fase condiciona a las siguientes y, a su vez, su inicio es influido por el desarrollo de las que le preceden. Cada fase es así interdependiente ya que las decisiones que se adopten en una, influyen sobre las que le siguen en el proceso. Por último, para su adecuado funcionamiento se requiere la existencia de buenos sistemas de información.

La planificación es la fase en la que se definen e identifican los objetivos últimos, entendidos como grandes metas o fines que la organización se fija de cara al futuro, se buscan alternativas o estrategias válidas para alcanzar dichas metas, entendiendo las alternativas como distintos caminos a través de los cuales se llega desde la situación actual hasta aquella que se trata de conseguir. En este momento se produce también el análisis de cada una de esas alternativas expresadas en forma de programas y finalmente se seleccionan aquellos programas que entendemos que mejor van a permitir alcanzar los objetivos.

Cada uno de los programas seleccionados para su ejecución deberá tener especificados objetivos concretos, claros y definidos. Igualmente, deberán tener señalados indicadores que permitan medir el grado de consecución de los objetivos. Sería muy conveniente que los indicadores escogidos permitieran conocer no sólo el grado de realización del programa, sino los efectos que su realización produce en la sociedad, es decir, debería tener indicadores no sólo de realización, sino de impacto, gestión, etc.

La presupuestación es la fase de concreción anual de la secuencia seguida hasta el momento. Conocidas las disponibilidades financieras para el próximo año, y dado que el presupuesto es un plan operativo y financiero para un año, tendremos que 
fijar para los programas elegidos en la planificación, las actividades a realizar y por tanto la parte de los objetivos concretos a conseguir en ese período de un año. La ejecución presupuestaria nos debe permitir reajustar nuestra actividad anual de manera que podamos conseguir el mejor de los resultados posibles con los medios que en cada momento podamos manejar.

Finalmente, en este proceso que tan escuetamente venimos exponiendo, el punto final lo pondrá la evaluación y control. El control del proceso es algo esencial a este sistema de gestión, cierra el modelo y permite su permanente mejora. Los controles ligados al sistema de gestión integrada deben permitir verificar que se ha realizado una gestión eficaz, eficiente, económica y, por supuesto, legal, ya que la Administración actúa sometida al imperio de la Ley.

Un sistema integrado de gestión implica y explica, tanto a nivel empresarial como gubernamental, el cambio producido en la contabilidad que ha evolucionado desde una contabilidad analítica estricta a una contabilidad de gestión con un contenido más amplio que facilita un mejor y más completo conocimiento de la realidad interna de la empresa de manera que el gestor pueda tomar decisiones más acertadas.

La eficiencia como se acaba de decir relaciona los resultados obtenidos con los medios utilizados para su consecución, y para medir esa relación resulta precisa una valoración monetaria de ambas realidades que permita conocer el coste de los medios que se utilizan. Conocido este coste las elecciones presupuestarias pueden ser hechas con criterios de racionalidad. El coste sólo puede ser facilitado con cierta precisión por una contabilidad analítica. Si la contabilidad analítica se refiere al cálculo de los costes y márgenes de la actividad, la contabilidad de gestión tiene un ámbito más amplio de estudio en el tiempo y en el espacio, ya que abarca también temas de planificación y presupuestación.

\section{El marco de la contabilidad pública de gestión}

\subsection{Concepto y objetivos de la contabilidad analitica pública}

La contabilidad analítica constituye, pues, un sistema de información que pretende determinar el coste de los productos obtenidos, analizar los resultados de la explotación y facilitar información para la toma de decisiones. Por costes, a su vez, podemos entender la medida y valoración de los consumos necesarios en la actividad productiva. Ambos conceptos: contabilidad analítica y coste son válidos tanto para las entidades lucrativas, como para las que no tienen ánimo de lucro, es decir, para las públicas. Conviene, sin embargo, una vez más, hacer referencia a las peculiares características de las Administraciones Públicas como entidades dedicadas a la producción de bienes y servicios para la colectividad y financiadas con pagos obligatorios de los ciudadanos, entidades en las que no rige la idea de beneficio que permite medir los resultados en las empresas privadas. Esta especial característica implica que en el ámbito público la contabilidad analítica no determina márgenes o beneficios, si bien no por ello es menos necesaria. La contabilidad analítica pública deberá permitir poner en relación los objetivos de actividad marcados al sujeto contable, con el coste en que se ha incurrido para cumplirlos, de manera que en cada momento se pueda hacer respecto a aquellos la mejor elección posible. La contabilidad analítica pública ha de permitir analizar los costes, valorar los servicios prestados y enjuiciar la gestión realizada aun cuando no exista el concepto de beneficio.

El sistema de contabilidad debe estar enfocado a la obtención de información, por lo que se debe diseñar en base a los posibles usuarios de la información que facilite. Aquí al igual que sucede en relación al resultado cabe mencionar una peculiaridad de lo público frente a lo privado. En el campo privado, la contabilidad financiera se diseña para la información de las personas ajenas a la empresa a las que pueda interesar, en tanto que la contabilidad analítica y la de gestión generan información para uso interno de la empresa.

En el campo público, por el contrario, la información elaborada ya por la contabilidad analítica ya por la financiera, tiene como destinatarios:

- Al Parlamento y otros órganos públicos.

- A los propios ciudadanos.

La información facilitada sirve para que los posibles destinatarios puedan tomar decisiones económicas, sociales y políticas y para conocer y evaluar el uso que la Administración hace de los recursos obtenidos coactivamente de los ciudadanos. Por lo que a los objetivos posibles de la contabilidad analítica pública hace referencia, hay que señalar, entre otros, los siguientes:

a) Valorar el coste de las actividades y de la prestación de los servicios públicos.

b) Apoyar la adopción de decisiones sobre la producción de bienes o prestación de servicios.

c) Valorar los activos generados por la actividad del sector público.

d) Analizar desde una perspectiva de eficiencia el empleo de los recursos públicos.

e) Proporcionar una información adecuada al gestor.

f) Facilitar la confección y evaluación de los Presupuestos. 
Estos objetivos son generales y genéricos, por lo que pueden existir otros específicos que dependerán de la naturaleza del ente al que la contabilidad se refiera y ello porque si algo está claro es la necesidad de la contabilidad analítica de acomodarse a la organización en la que se desarrolla y a su entorno, para ser útil y cumplir sus fines.

En línea con los objetivos que se han señalado se pueden concretar como fines de la contabilidad analítica los siguientes:

a) Lograr un efectivo control de gestión que facilite la toma de decisiones.

b) Poder fijar precios y tasas en base al coste real del servicio.

c) Facilitar la elaboración de un presupuesto y clarificar desde la perspectiva de la buena gestión financiera la utilización de los recursos.

d) Facilitar la presentación de información de gestión ante los usuarios.

Para cumplir estos fines es evidente que el subsistema de la contabilidad analítica se integrará en un sistema general más amplio que es en definitiva el sistema general de información del ente en cuestión. La relación entre contabilidad analítica y financiera se producirá a través de uno de los sistemas tradicionales: monista (radical o moderado) o dualista (radical o moderado).

\subsection{La contabilidad analitica y la medida de la eficiencia}

Con anterioridad se señalaron los procesos de modernización de la gestión financiera en los que se encuentran inmersos muchos países en el momento actual. Esta renovación de la gestión pasa por la consolidación de técnicas de presupuestación ligadas al señalamiento de metas u objetivos y la elección racional entre las diferentes alternativas posibles para la consecución de esos objetivos está ligada, sin duda, a la posibilidad de conocer el coste de las actividades que se realizan, no para determinar el posible beneficio de las mismas, pero sí para conocer si la gestión realizada fue eficaz, eficiente y económica, es decir, para conocer si los recursos utilizados lo han sido de la forma mejor desde el punto de vista estrictamente económico, y ello con independencia de que determinadas decisiones puedan tener que ser tomadas teniendo también en cuenta otros puntos de vista. En definitiva, dotan de racionalidad a la gestión financiera que pasa por el establecimiento de sistemas integrados de gestión como el que configura el presupuesto por programas.

La aplicación del sistema presupuestario de programas exige tanto en la empresa como en la Administración un adecuado sistema de información contable en el que se puedan basar las decisiones que se toman. Parte imprescindible de ese sistema es la contabilidad analítica, hasta el punto de que una de las razones de la quiebra de esta técnica presupuestaria en las Administraciones Públicas ha sido y es la carencia que estos órganos tienen de ese instrumento de conocimiento de los costes de su actividad. Hay que concluir, por tanto, que si queremos dar satisfacción a los ciudadanos, mejorar la gestión financiera pública y ser más eficaces y eficientes en esta gestión, necesitaremos, ineludiblemente, avanzar en el establecimiento de una contabilidad analítica que nos permita conocer lo que nos cuestan los medios que aplicamos a realizar las actividades públicas y ello aun cuando no podamos ni queramos determinar el beneficio dinerario de esa actividad. En efecto, difícilmente podremos seguir hablando de elección de alternativas con criterios de racionalidad si de verdad no sabemos cuál es el coste exacto de cada una de ellas. Difícilmente podremos saber el importe concreto de la subvención que un determinado servicio público debe recibir si no conocemos el coste real del servicio que presta. Cualquier decisión puede estar justificada, pero su elección para que sea auténticamente racional implica conocer su coste y este sólo puede ser facilitado por una contabilidad analítica. Su utilización, pues, en la Administración es absolutamente imprescindible, aunque las dificultades a vencer para su implantación hayan de ser aun muy grandes.

Por lo demás, una parte esencial de un sistema integrado de gestión la constituye un adecuado control que permita conocer las desviaciones que se producen entre los costes previstos y aquellos en que realmente se ha incurrido con objeto de tomar decisiones correctoras. Un adecuado control debe permitir conocer a cada centro productor de bienes y servicios, aunque no estén destinados a la venta, su situación presupuestaria real en cada momento y debe, en fin, permitir un correcto análisis de los resultados. Si se examina con detenimiento cuanto acabamos de señalar, hay que llegar a la conclusión de que tampoco es posible que el control realice adecuadamente ninguna de estas funciones si no dispone de herramientas apropiadas, y entre esas herramientas, necesariamente, tiene que encontrarse un adecuado sistema de contabilidad analítica que permita calcular los costes de los medios a utilizar y, con posterioridad, saber con exactitud los factores que realmente se aplican al proceso de producción de los bienes y servicios públicos. En definitiva, sin contabilidad analítica no se puede pensar en realizar la presupuestación de una manera que nos aproxime a cumplir con exactitud las exigencias de los ciudadanos en materia de gestión financiera. Podrán producirse aproximaciones más o menos voluntaristas y parciales a esa mejora de gestión, pero en el fondo siempre estarán lastradas por la imposibilidad de calcular el coste de los medios que consumimos para prestar los citados servicios al ciudadano. De igual modo debemos añadir que un sistema de contabilidad analítica por si solo, no es suficiente, aunque sea necesario, para conseguir aquella finalidad. Un sistema adecuado de contabilidad analítica es imprescindible, aun cuando la Administración no utilice la técnica de 
presupuestación por programas, pero incluso aunque se utilice, también es necesario para aquellas actividades de estricta regulación en las que resulta muy complicado fijar objetivos claros, ya que es la única aproximación posible al cálculo del coste de dicha actividad reguladora y un conocimiento del coste es necesario, dado que los recursos que la Administración maneja son escasos y las exigencias ciudadanas de gestión racional son cada vez más fuertes.

En definitiva, una contabilidad analítica se hace necesaria para cualquier actuación de la Administración que pretenda:

Determinar y cuantificar las actividades a realizar.

Cuantificar los recursos a emplear.

Conocer el coste de los servicios públicos prestados.

Pero con ser importante la contabilidad analítica, no puede ser considerada suficiente, en un paso más para conseguir una mejor gestión resulta necesario llegar a la contabilidad de gestión, ya que si bien en ocasiones se habla de contabilidad de costes o analítica y contabilidad de gestión como sinónimos, se ha de considerar a ésta como un todo más amplio del que aquélla es únicamente la parte que nos conduce a determinar el coste de nuestros productos o de nuestra actividad. Como hace la American Accounting Association, (A.A.A.) podemos definir la contabilidad de gestión como "aplicación de las técnicas y conceptos apropiados al procesar los datos económicos históricos y previstos de una entidad, con el fin de asistir a la dirección en el establecimiento de un plan en base a objetivos económicos racionales, y a tomar las decisiones más ordenadas de cara a la consecución de tales objetivos".

El crecimiento, pues, y el propio contenido de la Contabilidad de gestión, viene dado por las exigencias informativas para la toma de decisiones, relacionadas con los siguientes temas en una enumeración que no pretende ser exhaustiva:

- La organización del proceso productivo.

- La optimización de la capacidad existente.

- La utilización de los medios disponibles.

- El factor humano.

- El aprovisionamiento de los factores corrientes de la producción.

- La asignación de responsabilidades, etc.

Incluso en un paso más lá contabilidad (¿directiva?) debe estar en condiciones de permitir:

- Recoger información interna y externa para formular el diagnóstico de la empresá.

- Dar información que permita planificar y controlar la empresa.

- Participar en la valoración de la viabilidad de los proyectos y programas existentes.

\subsection{Caracteristicas de la información en la contabilidad analítica}

Como se ha reiterado, la contabilidad analítica es un sistema de información que pretende of recer información relevante para quienes deben gestionar.

En la Administración Pública el presupuesto es algo que el gestor prepara, que una vez aprobado por el Parlamento se vuelve rígido y que se ha de cumplir tal como es aprobado. Introducir en él cualquier modificación implica seguir un camino legal perfectamente regulado. Esta rigidez tiene una clara consecuencia en el ámbito de la contabilidad analítica. Los costes a utilizar son más relevantes a la hora de confeccionar el presupuesto que a la hora de su ejecución, si bien el seguimiento con datos reales sobre costes de la ejecución y el control del presupuesto aprobado permiten influir sobre el siguiente con decisiones basadas en hechos sólidos y no en meras intuiciones, aparte la posibilidad permanente de actuar sobre las desviaciones detectadas.

La Administración Pública al establecer la contabilidad analítica parte con otro condicionante. Para el proceso de planificar y por supuesto para controlar es mejor utilizar costes estándares o costes presupuestados que costes históricos, pero para que esa mayor utilidad sea aprovechada es preciso que aquellos estén rigurosamente calculados, lo que hoy no ocurre en la Administración, razón por la que parece más adecuado implantar el sistema con el cálculo de costes históricos al menos en un primer momento.

Pueden existir y de hecho existen en la práctica diferencias entre la información que se necesita y aquella de que se dispone, ello es debido (FERNÁndez RodríGuez, 1994) a la poca planificación de las necesidades de información, a la falta de concreción de los objetivos, a la falta de claridad organizativa y a la ausencia de un responsable que centralice las necesidades de información, así como al grado de agregación y claridad de los datos aportados. Conocer el proceso de decisión y los centros decisores son pasos importantes para definir el tipo de información necesaria.

\section{Una aproximación a la medida de la eficiencia}

\subsection{El proyecto C.A.N.O.A.}

Como primera mención al cálculo de costes en la gestión pública española hemos de mencionar la que hacia el Texto de la Ley General Presupuestaria al final de la década de los seten- 
ta. De una manera más concreta fue la ya citada Orden Ministerial de 20 de septiembre de 1983 (hoy expresamente derogada) la que al aprobar el grupo 9 del Plan de Contabilidad Pública relativo a la Contabilidad Analítica constituyó la primera regulación coherente y sistemática de un modelo de Contabilidad de Costes, dirigido a los entes de carácter administrativo de las Administraciones Públicas.

Una resolución de la I.G.A.E. de 26 de diciembre de 1983 regulaba la forma de realizar determinados informes sobre los costes de funcionamiento de los diferentes servicios a rendir por los Departamentos Ministeriales. Para el cumplimiento de esta resolución la I.G.A.E. requirió información a los distintos Departamentos, pero únicamente algunos remitieron dicha información. La mejor conclusión a la que permitió llegar el tratamiento por la Subdirección General de Dirección y Planificación de la Contabilidad de la I.G.A.E. de la información recibida, fue la constatación del hecho de que, dada la enorme variedad de actividades y la complejidad organizativa de la Administración, no resultaba posible la implantación de un único sistema simple de cálculo de costes, y que para poder poner en marcha una contabilidad analítica era más sencillo hacerlo en la Administración Institucional, que en la Administración General, tanto por la mayor concreción de sus operaciones y por tanto la mayor precisión de los posibles centros de imputación, cuanto por el carácter de sus actividades muy próximas en algunas ocasiones al sector empresarial. Esta decisión ha dado lugar al proyecto desarrollado por la I.G.A.E. denominado CANOA (Contabilidad Analítica Normalizada de Organismos Autónomos).

El proyecto se ha diseñado durante los años 1993 y 1994. Durante 1995 se llevaron a cabo algunas experiencias piloto y durante 1996 debería desarrollarse la aplicación informática del sistema, de manera que en 1997 se estuviera en condiciones de implantarlo en todos los organismos que voluntariamente se han adherido a él.

El proyecto CANOA aparece como un subsistema de contabilidad analítica que se apoya en otros subsistemas de información. Para que el modelo tenga la operatividad necesaria, como mínimo requiere la existencia de los siguientes subsistemas:

- Gestión de personal

- Gestión de inventario

- Gestión de justificante de gastos

- Gestión de proyectos de inversión.

El sistema está diseñado para que los datos que la contabilidad precisa y que sean necesarios en alguno de estos subsistemas y en el de costes sean tomados de aquellos informáticamente en el momento que se determine, para pasar a bases de datos que permitan su tratamiento posterior separado.

El sistema de contabilidad analítica es así independiente de la contabilidad financiera, es decir, se trata de un modelo dualista radical.

El proyecto C.A.N.O.A. utiliza en su aplicación el cálculo de costes históricos o reales y ello porque, como antes señalaba, no existe experiencia en el campo público que permita recurrir a los costes estándares, aunque aspira a que éstos puedan ser utilizados más adelante cuando se pueda disponer de series históricas de mejor conocimiento de la realidad. En lo que se refiere al cálculo mismo del coste el proyecto se inclina por la técnica de utilización del coste completo (Full Costing). Aparte estas líneas generales, el modelo pretende ser flexible de manera que se debe personalizar en función de las características del ente al que se desee aplicar y de la actividad a la que se dedique dicho ente.

El modelo básico o general señala como base de suministro de datos para cálculo de los costes, todos los que afecten al organismo, cualquiera sea el lugar en que se produzcan, así distingue entre:

a) Costes procedentes de la ejecución de gastos del propio organismo.

b) Costes procedentes de la ejecución de los gastos de otros entes públicos.

c) Costes procedentes de cálculos internos del organismo.

Una vez recibidos los datos por el sistema hay que separar entre los que suponen cargas incorporables y que van a ser trasladados como costes, de los que son cargas no incorporables.

En las cargas incorporables y que se trasladan como costes de las actividades del organismo, se distinguen entre costes externos y costes calculados. Entre los costes externos podemos incluir los de personal, adquisición de bienes corrientes y servicios y el coste de los almacenes. Entre los costes calculados se incluyen la amortización y la previsión social de los funcionarios. En un primer momento en el proyecto CANOA no se han considerado las diferencias de inventario ni otros costes calculados, en especial los de oportunidad.

Como destino de los costes u objeto de coste el proyecto escoge a las actividades del organismo o a los centros de coste, si bien recomienda, creo que acertadamente, siempre que sea posible, imputar directamente costes a las actividades del organismo, lo que no implica dejar de calcular el de los diversos centros, lo que resulta lógico, pues el objetivo básico es el cálculo del coste de la actividad o del servicio prestado, aunque igualmente debe conocerse el coste de los lugares físicos donde éste se produce.

Los centros de coste para poder ser considerados como tales deben reunir una serie de condiciones, entre ellas:

a) Que sean unidades orgánicas diferenciadas. 
b) Que realicen actividades concretas, que se materializarán normalmente en servicios públicos prestados.

c) Que exista un responsable de centro.

Entre las actividades el proyecto distingue entre actividades finalistas y actividades intermedias, en función de que reciban sus propios costes y parte de los de otras actividades, o de que repartan los costes entre las actividades finalistas. Son, pues, las actividades finalistas las que concretan los productos o resultados finales de la actividad realizada.

El proyecto CANOA termina con el cálculo de los costes, pues en lo que se refiere a los ingresos, éstos son obligatorios y afluyen al ente sin relación, normalmente, con la actividad que presta. Si se trata de un organismo con ingresos propios ligados a la actividad que realiza, como es el caso de algunos de la Administración Institucional a la que el proyecto se aplica, éste debe servir de base para el cálculo de los precios y tasas a establecer por los servicios que presta a los ciudadanos, tal y como prevé la Ley de Tasas y Precios Públicos. En este caso, como ingresos se incluirán subvenciones corrientes, tasas, precios públicos, precios privados, multas, sanciones, ingresos diversos, etc. Todos los ingresos que se puedan relacionar con actividades finalistas concretas se compararán con el coste de dichas actividades. Si no se pueden imputar a actividades concretas, no se compararán con costes globales. Así, el grado de cobertura de la actividad lo dará la comparación entre ingresos y costes de las actividades finalistas.

Como es lógico, el sistema de contabilidad analítica examinado se diseña para producir información útil para la toma de decisiones y para mejor gestionar lo público, por tanto, se proyecta para producir información que debe procurar ser lo más adecuada posible, sin sesgos y que incorpore tanto series numéricas como su explicación. La información a suministrar por el sistema puede ser: intermedia y final.

La intermedia está destinada a la dirección del ente. La final aunque destinada a la dirección, tiene otros destinatarios, entre ellos los órganos de control y los propios ciudadanos. Es precisamente ese destino final de la información producida el que una vez más pone la nota diferenciadora entre la contabilidad analítica pública y la privada, pues en ésta siempre serán destinatarios de la información los gestores de la empresa, en tanto en aquella lo seremos también los ciudadanos.

Para terminar, decir que se trata de una experiencia que intenta introducir en la Administración Pública, una aproximación a una gestión cada día más eficiente de los recursos públi$\cos$. Si los proyectos requieren un momento adecuado y medios idóneos para tener éxito, éste, sin duda lo tendrá, ya que el momento es adecuado, porque más que nunca, hoy, el ciudadano exige de la Administración Pública una buena gestión financiera, que no tiene asegurada por el hecho de que se disponga de un sistema de contabilidad analítica, pero que es mucho más difícil sin él, y además dispone de medios idóneos, ya que el desarrollo informático actual permite tratamientos complejos, que eran impensables hace relativamente pocos años.

El proyecto CANOA, cuyas líneas generales se acaban de comentar, ha sido experimentado por la Intervención General en varios Organismos Autónomos y ha permitido llegar a la determinación manual de sus costes. Obviamente, para que el sistema pueda ser aplicado masivamente deberá ser realizado su desarrollo informático por la Dirección General de Informática Presupuestaria (DGIP). Si este desarrollo se realiza como está previsto y es deseable durante 1996, sólo quedará su aplicación a partir de 1997 y el deseo de que con ello se pueda dar un importante paso en la mejora de la gestión económico-financiera pública y, con ella, en el funcionamiento general de la Administración.

El camino, pues, está ahí, nuestra obligación es recorrerlo para poder gestionar más eficientemente en beneficio de todos los ciudadanos. Sabemos que puede ser largo y complicado, pero en la medida en que se dispone de conocimientos y medios ha de ser abordado, ya que, como dijo el poeta, el camino son únicamente nuestras huellas.
- Subdirector General de Estudios y Coordinación de la Intervención General de la Administración del Estado.

${ }^{1}$ El presente trabajo, ahora retocado, constituye la base de la conferencia que su autor pronunció en la Universidad de Lima, dentro del Seminario Costes Estratégicos y Gestión Empresarial celebrado en dicha ciudad los dias 29 y 30 de noviembre y 1 de diciembre de 1995 . 


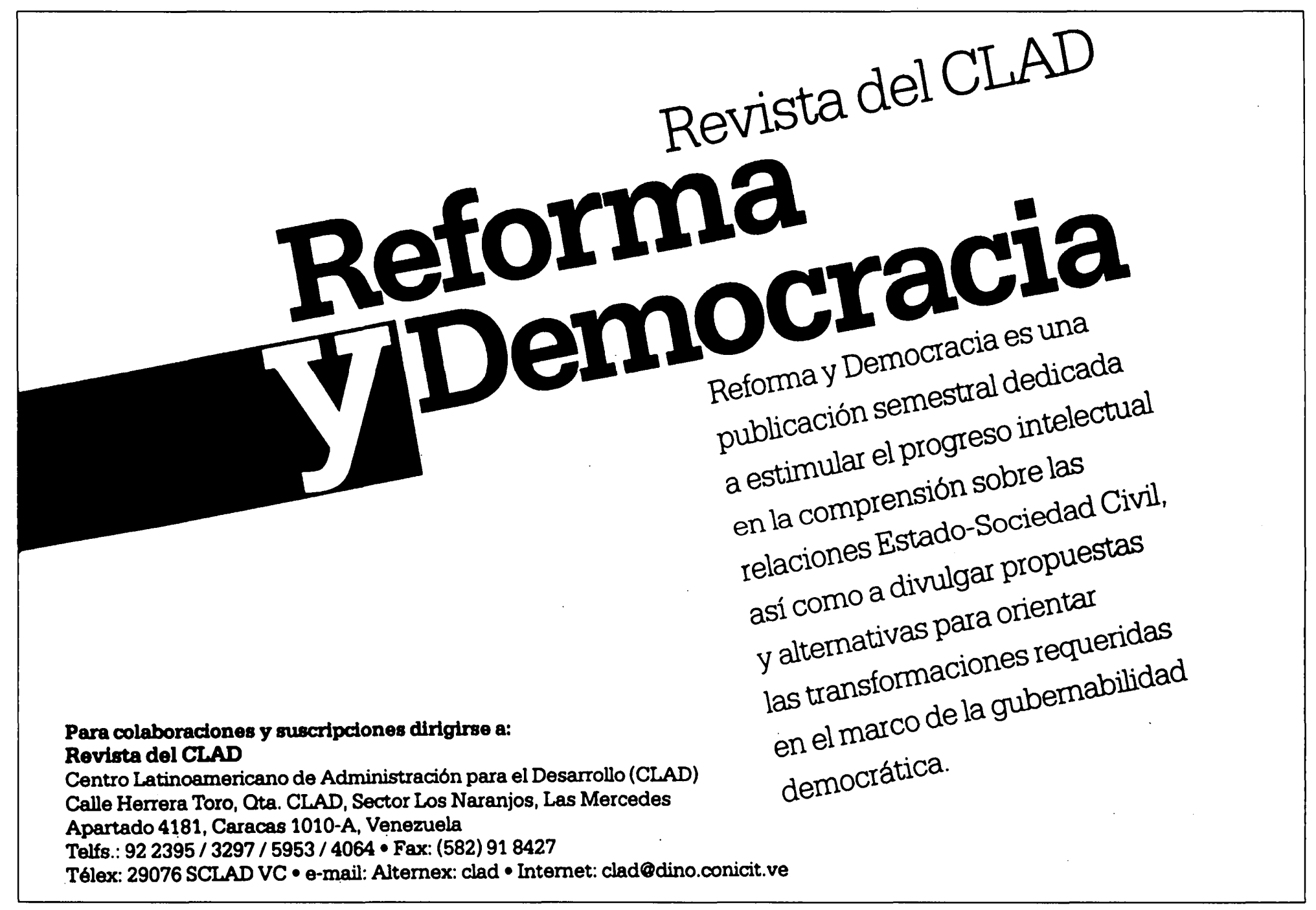

